\title{
Éditorial
}

\section{Le flux et le reflux}

\author{
Philippe Lesclous
}

L'histoire des recommandations de bonne pratique en matière médicale est tel l'océan, tempétueuse parfois, encore trop souvent énigmatique et soumise à des mouvements de flux et de reflux, reflets des limites de leur genèse. Ainsi, l'histoire de l'antibioprophylaxie de l'endocardite infectieuse (ABP EI) dans notre domaine professionnel connaît une nouvelle période, difficilement compréhensible comme les autres pour un esprit cartésien.

Inaugurée « officiellement » par l'American Heart Association en 1955 [1], l'ABP EI a connu une phase rapidement expansive et radicale jusqu'en 2002, année au cours de laquelle une phase descendante est amorcée par un référentiel français [2]. Le mouvement s'est ensuite accentué en 2008, allant même jusqu'à la suppression radicale de toute ABP EI systématique par un groupe d'experts britanniques du National Institute of Health and Clinical Excellence [3]. Tout cela sans preuve scientifique formelle de son efficacité comme de son inefficacité [4]. Aujourd'hui, dans le monde entier, sauf chez les Britanniques donc, l'ABP EI est restreinte aux procédures dentaires entraînant une bactériémie jugée significative chez les patients à haut risque $\mathrm{d}^{\prime} \mathrm{EI}[5,6]$.

Cette notion de procédure dentaire entraînant une bactériémie jugée significative est très aléatoire puisque, dans la plupart des cas d'EI, il n'existe pas de données bactériologiques probantes. Et pourtant, tout dernièrement, la Société européenne de cardiologie (ESC), au décours de la révision de ses recommandations de bonne pratique, vient de jeter un pavé dans la mare en stipulant que désormais il n'y avait aucune évidence scientifique à continuer d'interdire la pose d'implants dentaires chez les patients à haut risque d'EI (à condition d'un suivi régulier de chaque patient) [7]. Pour aussi séduisante qu'elle paraisse, cette recommandation repose sur la foi d'une série de 13 patients (57 implants) sur une période de 17 ans [8] ! L'ESC demande à chaque pays de faire valider cette révision par les autorités des pays membres, dont la France.

La Société française de cardiologie, la Société française de parodontologie et d'implantologie orale et la Société française de chirurgie orale ont décidé d'unir leurs efforts en constituant un groupe de travail commun (dirigé par un membre de la SFCO, le docteur Sarah Millot, MCU-PH à l'UFR d'odontologie de Paris Descartes) dont l'objectif est de fournir un cadre à la mise en œuvre de cette recommandation de l'ESC. Ce groupe, bien conscient des limites de l'ouverture de cette pratique implantaire à cette catégorie de patients, a décidé d'orienter son travail selon plusieurs axes:

- établir des règles concernant le cadre chirurgical de la mise en place implantai re en prenant en compte le sur-risque infectieux potentiel immédiat chez des patients à haut risque d'EI ; - tenir compte des facteurs de risque identifiés ou fortement suspectés comme aggravant l'échec implantaire ou le risque infectieux de manière à minimiser au maximum le risque de péri-inhalent-ils. Ces facteurs sont liés au patient (habitude de vie, antécédents locaux et généraux...) ainsi qu'à l'implant lui-même (état de surface...);

- et finalement proposer un cahier des charges implantaires chez ces patients à haut risque d'EI.

On ne peut que louer cette volonté d'unir des compétences et des efforts. On ne peut qu'appeler à la réussite et à la plus grande diffusion des travaux de ce groupe. On ne peut que se réjouir que la SFCO soit motrice dans ce processus.

Mais une question demeure, comment une société médicale scientifique, l'ESC en l'occurrence, peut-elle décider de faire évoluer des recommandations sur la foi d'un rapport de cas clinique ? Une énigme, je vous dis, qui illustre bien les limites actuelles de la réalisation de ce type de recommandations.

\footnotetext{
* Correspondance : philippe.lesclous@univ-nantes.fr

This is an 0pen Access article distributed under the terms of the Creative Commons Attribution License (http://creativecommons.org/licenses/by/4.0), which permits unrestricted use, distribution, and reproduction in any medium, provided the original work is properly cited
} 


\section{Références}

1. Committe on prevention on rheumatic fever and bacterial endocarditis. Prevention on rheumatic fever and bacterial endocarditis through streptococcal infections. Circulation 1955; 11:317-320.

2. Société de Pathologie Infectieuse de Langue Française, Société Française de Cardiologie. Révision de la conférence de consensus de la prophylaxie de l'endocardite infectieuse. Argumentaire. Med Mal Infectieuse 2002;32:562-595.

3. National Institute of Health and Clinical Excellence. Prophylaxis against infective endocarditis. Centre for Clinical Practice at Nice (UK) 2008.

4. Dayer MJ, Jones S, Prendergast B, Baddour LM, Lockhart PB, Thornhill MH. Incidence of infective endocarditis in England
2002-13: a secular trend, interrupted time-series analysis. Lancet 2015;385:1219-1228.

5. Agence Française de Sécurité Sanitaire et des Produits de Santé. Prescription des antibiotiques en pratique bucco-dentaire: recommandations de bonne pratique. Med Mal Infect 2011;42:193-212.

6. Cloitre A, DuvalX, Lesclous P. Antibioprophylaxie de l'endocardite infectieuse, respect des recommandations de bonne pratique, enquête nationale auprès des chirurgiens dentistes. Med Buc Chir Buc 2014;20:141-153.

7. Habib G. et al. 2015 ESC guidelines for the management of infective endocarditis. The task force for the management of infective endocarditis of the European Society of Cardiology (ESC). Eur Heart J 2015;36:3075-3128.

8. Findler M, Chackhartchi T, Regev E. Dental implants in patients at high risk for infective endocarditis: a preliminary study. Int J Oral Maxillofac Surg 2014;43:1282-1285. 